\title{
Article \\ Adaptive Robust Position Control of Electro-Hydraulic Servo Systems with Large Uncertainties and Disturbances
}

\author{
Manh Hung Nguyen (D), Hoang Vu Dao (D) and Kyoung Kwan Ahn *(D) \\ School of Mechanical and Automotive Engineering, University of Ulsan, Daehakro 93, Namgu, \\ Ulsan 44610, Korea; hungnguyen@ulsan.ac.kr (M.H.N.); daohoangvu95@ulsan.ac.kr (H.V.D.) \\ * Correspondence: kkahn@ulsan.ac.kr
}

check for updates

Citation: Nguyen, M.H.; Dao, H.V.; Ahn, K.K. Adaptive Robust Position Control of Electro-Hydraulic Servo

Systems with Large Uncertainties and Disturbances. Appl. Sci. 2022, 12, 794. https://doi.org/10.3390/ app12020794

Academic Editor: Jeong Ik Lee

Received: 12 December 2021

Accepted: 10 January 2022

Published: 13 January 2022

Publisher's Note: MDPI stays neutral with regard to jurisdictional claims in published maps and institutional affiliations.

Copyright: (C) 2022 by the authors. Licensee MDPI, Basel, Switzerland. This article is an open access article distributed under the terms and conditions of the Creative Commons Attribution (CC BY) license (https:// creativecommons.org/licenses/by/ $4.0 /)$.

\begin{abstract}
In this paper, a novel adaptive robust control (ARC) scheme is proposed for electrohydraulic servo systems (EHSSs) with uncertainties and disturbances. All dynamic functions in system dynamics are effectively approximated by multi-layer radial basis function neural network (RBF NN)-based approximators with online adaptive mechanisms. Moreover, neural network-based disturbance observers (NN-DOBs) are established to actively estimate and efficiently compensate for the effects of not only the matched/mismatched but also the imperfections of RBF NN-based approximators on the control system. Based on that, the nonlinear robust control law which integrates RBF NNs and NN-DOBs is synthesized via the sliding mode control (SMC) approach to guarantee the high-accuracy position tracking performance of the overall control system. Furthermore, the problem of the combination between DOBs and RBF NNs is first introduced in this paper to treat both disturbances and uncertainties in the EHSS. The stability of the recommended control mechanism is proven by using Lyapunov theory. Finally, numerical simulations with several distinct frequency levels of reference trajectory are conducted to convincingly demonstrate the effectiveness of the proposed approach.
\end{abstract}

Keywords: electro-hydraulic servo system (EHSS); RBF-neural network (RBF-NN); sliding mode control (SMC); disturbance observer (DOB); high-order sliding mode Levant's differentiators

\section{Introduction}

Hydraulic servomechanisms have been broadly employed in various industrial appli cations-e.g., hydraulic robot manipulators [1], hydraulic press [2], load simulators [3], vehicle active suspension systems [4], and so on-due to their superiorities such as a high power-to-weight ratio, fast and smooth response, high stiffness, and ability to generate a tremendous force/torque [5]. However, the inherent highly nonlinear characteristics, parameter variations, modeling uncertainties, and external disturbances of the EHSS are still the major obstacles to achieving high-accuracy tracking performance. Hence, the position tracking problem of the EHSS has attracted many studies in recent years.

To control EHSSs, proportional-integral-derivative (PID) control law [6] was initially employed due to its simple structure and easy implementation. However, it is difficult to achieve satisfactory performance since the system dynamics are not compensated in the control law. Alternatively, model-based control strategies for EHSSs such as feedback linearization control (FLC) [7], backstepping control (BC) [8], and adaptive control (ADC) [9] have been widely employed to achieve a better tracking performance. Although in these control methods, the system dynamics are taken into account in control design, they are sensitive to the modeling uncertainties and disturbances that naturally exist in EHSSs. In contrast, SMC [10-14] which was originally introduced by Utkin [15], is considered as a powerful candidate to deal with disturbances and uncertainties, satisfying the matching condition in nonlinear systems that are assumed to be bounded. However, it can be seen that all the above model-based control approaches for EHSSs require the system 
dynamics to be known, which are complicated and difficult to precisely acquire due to high nonlinearities in pressure dynamics and a number of unknown parameters that need to be identified from experimental input-output data [16].

By virtue of the excellent properties of their self-learning capability and online weightadaptive mechanisms, radial basis function (RBF) neural networks (NNs) have been employed in various fields such as robot manipulators [17-20], magnetic levitation systems [21], aircraft [22], hydraulic systems [5,23-26], and so on to approximate the unknown dynamic components of control systems. For EHSSs, Z. Yao et al. introduced multilayer RBF NNs to approximate partly mismatched and matched uncertainties [5], and semiglobal asymptotic stability was achieved accordingly. In [23], an NN-based estimator was proposed to approximate an unknown term generated in the control design of the hydraulic active suspension systems (ASSs). Besides, an RBF NN-based function approximator [24] was developed to compensate for the effects of the nonlinear friction in EHSSs. In [25], parametric uncertainties and an unknown external load in hydraulic systems were grouped into so-called generalized uncertainties, which were compensated by using RBF NNs. However, in the above-mentioned works, RBF NNs were only exploited to partially approximate model uncertainty in the control system. Hence, the employment of RBF NNs for approximating full model uncertainties is still an open problem.

Besides, although RBF NNs are capable of dealing with model uncertainties, they cannot effectively treat disturbances whose behaviors are independent of the system states. Hence, the employment of disturbance observers (DOBs) can be considered as an effective way to cope with disturbances in EHSSs. Extended state observers (ESOs) were employed $[27,28]$ for EHSSs to estimate both unmeasurable system states and matched/ mismatched disturbances; therefore, high-accuracy tracking performances were achieved. In another approach to cope with disturbances, some DOB designs based on exact differentiators [29] have been applied in hydraulic systems. For example, a linear disturbance observer (LDOB) [30] for hydraulic rotary actuator (HRA) control systems and nonlinear disturbance observers (NDOBs) [1,31] for hydraulic robot manipulators have been successfully employed to actively reject the influence of the grouped disturbances on the tracking performance. It is noted that the above-mentioned DOB designs require the construction of an observer with a careful tuning process of observer gains to achieve highly efficient disturbance compensation. Hence, to simplify the parameter tuning, the unknown disturbance estimator (UDE) [32] has been proposed with only one parameter that needs to be selected. Nevertheless, similar to model-based control approaches, DOB designs require system dynamic information, which inhibits the applications of DOBs. In addition, the employment of RBF-NNs and DOBs to cope with partial uncertainties and disturbances has been studied [33,34]. However, to the best of the authors' knowledge, the integration of RBF NNs and DOBs into a controller to treat the problem of full model uncertainties and disturbances is still challenging, and this is considered in this paper.

Motivated by the above comprehensive analysis, in this paper, a novel adaptive robust control mechanism for the EHSS with completely unknown dynamics and disturbances is originally proposed. In this framework, high-order sliding mode exact differentiators [35] are adopted to exactly calculate the angular velocity, acceleration, and pressure derivative. Based on that, all unknown dynamic functions in system dynamics are approximated by RBF-NN-based approximators. For the first time, a pair of NN-based DOBs is established to estimate and compensate for the effects of the NN approximation imperfections, unmodeled dynamics, and external load on the control system of the EHSS simultaneously. Finally, the full-state feedback robust control law is synthesized to ensure the high-accuracy tracking performance in the presence of completely unknown dynamic functions and both matched/mismatched lumped disturbances. The overall system stability is proven by utilizing the Lyapunov theory. Several simulations are conducted to verify the effectiveness of the proposed control strategy.

The main contributions of this paper are summarized as follows: 
1. The RBF NN-based function approximators with adaptive mechanisms are designed to online approximate all unknown dynamic functions in the dynamics of the EHSS.

2. A pair of DOBs based on the HOSM differentiators along with NNs are first developed to effectively estimate and actively compensate for not only the effects of both mismatched and matched disturbances but also the imperfections of the function approximation of RBF NNs.

3. A robust control law based on the RBF NNs and DOBs is synthesized to guarantee the high-accuracy tracking performance of the EHSS control system under the impacts of large model uncertainties and disturbances.

4. The combination of RBF NNs and DOBs in order to take all their advantages is originally introduced to efficiently treat both full model uncertainties and disturbances in the dynamics of EHSSs.

The remainder of the paper is organized as follows. In Section 2, the system modeling is presented. The high-order differentiator, neural network-based system dynamic approximators, disturbance observers, and control strategy are developed in Section 3. The total system stability is analyzed in Section 4 and comparative numerical simulations are conducted in Section 5. Finally, Section 6 concludes this paper.

\section{System Modeling}

The structure of the studied EHSS is presented in Figure 1. In this configuration, the angular position and pressures of the hydraulic rotary actuator chambers are measured by encoder and pressure transducers, respectively. To control the fluid flow in/out of the actuator, a high-bandwidth servo valve is utilized. The problem is how to design a control law such that the angular position of the HRA tracks the reference trajectory as closely as possible in the presence of both mismatched and matched disturbances and completely unknown system dynamic functions with the assumption that the radian displacement per revolution of the HRA is determined by manufacturer's datasheets and the moment of inertial of the load is known.

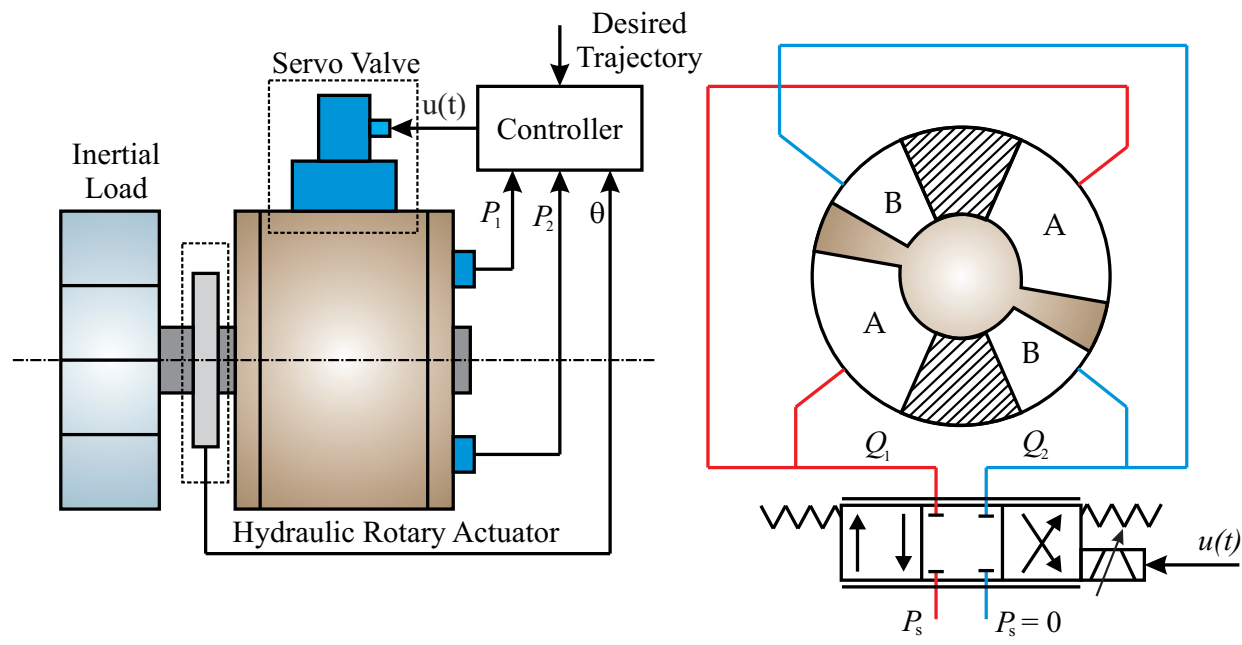

Figure 1. The structure of the studied servo valve-controlled electro-hydraulic system.

To carefully investigate the overall system dynamics, the system is divided into two subsystems; i.e., mechanical and hydraulic systems. The dynamics of these subsystems are presented in the following sections.

\subsection{Mechanical System}

The motion dynamics of the inertial load are obtained by applying Newton's second law as

$$
J \ddot{\theta}=\tau_{L}-\tau_{f}(\dot{\theta})-\tau_{d}
$$


where $J$ and $\theta$ are the moment of inertia and the angular position of the load, respectively. $\tau_{L}$, $\tau_{f}$, and $\tau_{d}$ are the load torque of the hydraulic actuator, frictional torque, and disturbances caused by modeling errors, unmodeled dynamics, and external load, respectively.

The load torque generated by the pressure difference between chambers of the actuator is determined by [36]

$$
\tau_{L}=P_{L} D_{m}
$$

where $P_{L}=P_{1}-P_{2}$ is the load pressure with $P_{1}$ and $P_{2}$ representing the pressures of the two actuator chambers and $D_{m}$ denotes the radian displacement of the actuator.

Due to the high stiffness of EHSSs, the frictional torque can be modeled without considering its dynamics as follows [16]:

$$
\tau_{f}=B \dot{\theta}+\left(\tau_{c 0}+\tau_{s 0} \exp \left(-\frac{|\dot{\theta}|}{\omega_{s}}\right)\right) \operatorname{sgn}(\dot{\theta})
$$

where $B$ is the viscous friction coefficient, $\tau_{c 0}$ and $\tau_{s 0}$ are the parameters for Coulomb and Stribeck frictions, $\operatorname{sgn}(\bullet)$ and $\exp (\bullet)$ represent the standard signum and exponential functions, respectively, and $\omega_{s}$ is Stribeck velocity. Consequently, the frictional torque in (3) can be re-arranged as

$$
\begin{aligned}
& \tau_{f}=\tau_{s m f}+\Delta \tau_{f} \\
& \tau_{s m f}=B \dot{\theta}+\left(\tau_{c 0}+\tau_{s 0} \exp \left(-\frac{|\dot{\theta}|}{\omega_{s}}\right)\right) \tanh \left(c_{f} \dot{\theta}\right)
\end{aligned}
$$

where $\tau_{s m f}$ represents the smoothened term of frictional torque, $\tanh (\bullet)$ is the hyperbolic tangent function, $c_{f}$ is a positive constant, and $\Delta \tau_{f}$ denotes the approximation error caused by replacing the $\operatorname{sgn}(\bullet)$ function by the $\tanh (\bullet)$ function.

Combining (1), (2) and (4), one obtains

$$
\ddot{\theta}=\frac{D_{m}}{J} P_{L}-\frac{\tau_{s m f}}{J}-\frac{\Delta \tau_{f}+\tau_{d}}{J}
$$

\subsection{Hydraulic System}

Due to the development of hydraulic seals, it is assumed that the external leakage is negligible and can be ignored, and the load pressure dynamic can be described as [36]

$$
\frac{V_{t}}{4 \beta_{e}} \dot{P}_{L}=-D_{m} \dot{\theta}-q_{L}+Q_{L}+q(t)
$$

where $V_{t}$ represents the whole control volume of the actuator, $\beta_{e}$ is the bulk modulus of the hydraulic oil, $q_{L}$ denotes the internal leakage caused by the pressure discrepancy between the chambers of the actuator, $Q_{L}$ signifies the load flow, and $q(t)$ is the modeling uncertainties.

The internal leakage model is given by [37]

$$
q_{L}=c_{1} P_{L}^{2}+c_{2} P_{L}+c_{3}
$$

where $c_{1}, c_{2}$, and $c_{3}$ are constants.

The load flow is computed by [16]

$$
Q_{L}=C_{d} w x_{v} \sqrt{\frac{1}{\rho}\left(P_{s}-\operatorname{sgn}\left(x_{v}\right) P_{L}\right)}
$$

where $C_{d}$ denotes the discharge coefficient, $w$ is the spool valve area gradient, $\rho$ is the oil density, and $P_{S}$ represents the supply pressure.

Since the tracking performance is only slightly improved and the system order increases significantly if the full dynamics of the servo valve are considered [38], to reduce 
the complexity of control design, a simplified model in which valve spool displacement is directly proportional to the control input can be used as

$$
x_{v}=k_{v} u
$$

where $x_{v}$ signifies the spool displacement, $k_{v}$ represents the servo valve coefficient, which is determined based on its datasheet provided by the manufacturer, and $u$ is the control input voltage.

Based on (8) and (9), one obtains

$$
Q_{L}=k_{t} u \sqrt{\left(P_{s}-\tanh \left(c_{v} u\right) P_{L}\right)}+\Delta Q_{L}
$$

where $k_{t}=C_{d} w k_{v} \sqrt{1 / \rho}$ denotes the total load flow coefficient, $c_{v}$ is a positive constant, and $\Delta Q_{L}$ represents the approximation error when smoothing the load flow Equation (8).

\subsection{Total System}

Defining $\mathbf{x}=\left[x_{1}, x_{2}, x_{3}\right]^{T}:=\left[\theta, \dot{\theta}, D_{m} P_{L} / J\right]^{T}$ as the state vector, based on (4)-(7) and (10), the total system dynamics can be derived in a strict feedback form as

$$
\begin{aligned}
& \dot{x}_{1}=x_{2} \\
& \dot{x}_{2}=x_{3}+f_{1}(\mathbf{x})+d_{1}(\mathbf{x}, t) \\
& \dot{x}_{3}=f_{2}(\mathbf{x})+g_{2}(\mathbf{x}, u) u+d_{2}(\mathbf{x}, t)
\end{aligned}
$$

where $f_{1}(\mathbf{x}), d_{1}(\mathbf{x}, t), f_{2}(\mathbf{x}), g_{2}(\mathbf{x}, u), g_{2}(\mathbf{x}, u)$ and $d_{2}(\mathbf{x}, t)$ are given by

$$
\begin{aligned}
& f_{1}(\mathbf{x})=-\frac{B}{J} x_{2}-\frac{1}{J}\left(\tau_{c 0}+\tau_{s 0} \exp \left(-\frac{\left|x_{2}\right|}{\omega_{s}}\right)\right) \tanh \left(c_{f} x_{2}\right) \\
& d_{1}(\mathbf{x}, t)=-\frac{\Delta \tau_{f}+\tau_{d}}{J} \\
& f_{2}(\mathbf{x})=-\frac{4 \beta_{e} D_{m}^{2}}{J V_{t}} x_{2}-\frac{4 \beta_{e}}{V_{t}}\left(c_{1} \frac{J^{2}}{D_{m}^{2}} x_{3}^{2}+c_{2} \frac{J}{D_{m}} x_{3}+c_{3}\right) \\
& g_{2}(\mathbf{x}, u)=\frac{4 \beta_{e} k_{t}}{V_{t}} \sqrt{P_{s}-\tanh \left(c_{v} u\right) \frac{J}{D_{m}} x_{3}} \\
& d_{2}(\mathbf{x}, t)=\frac{4 \beta_{e}}{V_{t}}\left(\Delta Q_{L}+q(t)\right)
\end{aligned}
$$

To facilitate the controller design, some reasonable assumptions are made as follows:

Assumption 1. The desired trajectory $x_{1 d}(t)$ is sufficiently smooth, and its derivatives up to order three are bounded.

Assumption 2. The pressures of the actuator chambers $P_{1}$ and $P_{2}$ are bounded by the supply pressure $P_{s}$. The absolute value of the load pressure $P_{L}$ is adequately smaller than the supply pressure $P_{s}$ to ensure the function $g_{2}(\mathbf{x}, u)$ is distant from zero.

Assumption 3. The mismatched and matched lumped disturbances $d_{1}(\mathbf{x}, t)$ and $d_{2}(\mathbf{x}, t)$ are smooth enough and their first-order derivatives are bounded by positive constants; i.e., $\left|\dot{d}_{1}(\mathbf{x}, t)\right| \leq \delta_{1}$ and $\left|\dot{d}_{2}(\mathbf{x}, t)\right| \leq \delta_{2}$.

\section{Adaptive Robust Control Design}

The control scheme of the proposed method is depicted in Figure 2. In this configuration, the angular velocity, angular acceleration, and the first-order derivative of the load pressure are determined by employing Levant's high-order exact differentiators. Then, the 
unknown dynamic functions-i.e., $f_{1}(\mathbf{x}), f_{2}(\mathbf{x})$, and $g_{2}(\mathbf{x}, u)$ - caused by unknown system parameters are approximated based on RBF NN-based approximators. A pair of NN-based DOBs are designed to estimate not only mismatched/matched lumped disturbances but also approximation errors of RBF NNs. Finally, a control law is synthesized to guarantee the stability of the overall system and achieve high-accuracy tracking performance.

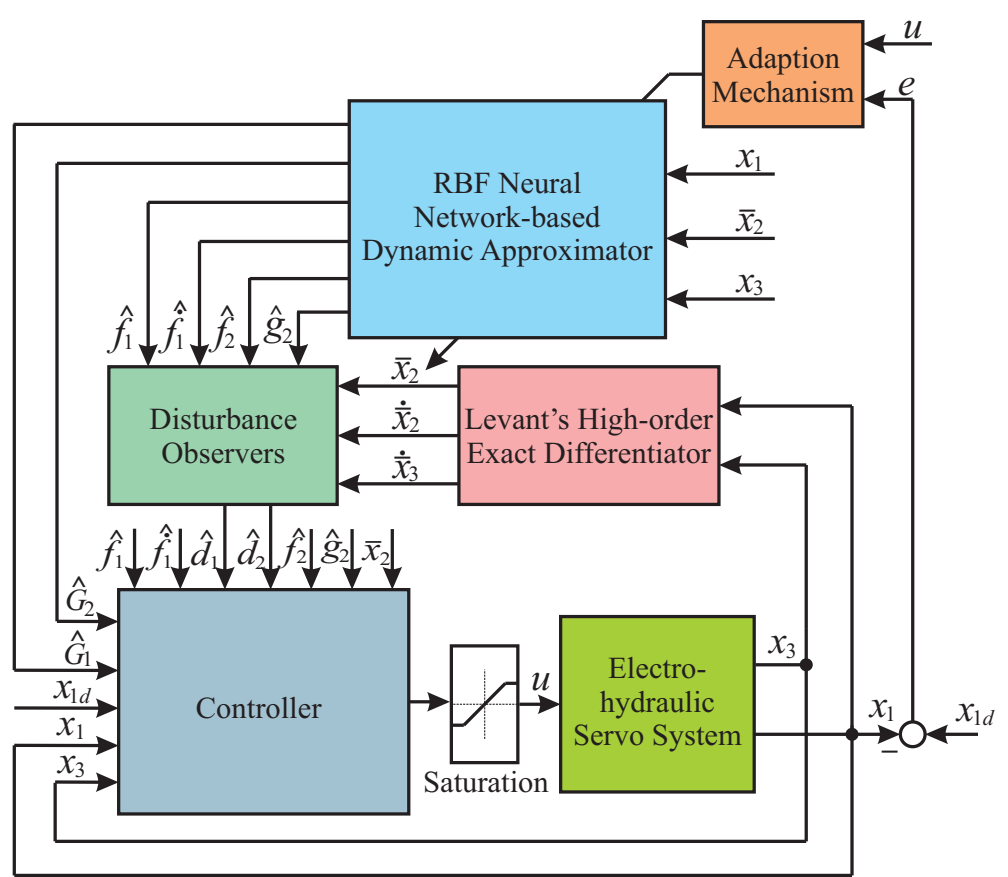

Figure 2. The control scheme of the proposed control strategy.

\subsection{Robust Control Law Design}

To facilitate the controller design, it is assumed that the system dynamic functions $f_{1}(\mathbf{x}), f_{2}(\mathbf{x})$, and $g_{2}(\mathbf{x}, u)$, which are approximated later, are completely known. In addition, the mismatched/matched lumped disturbances and angular velocity of the inertial load are measurable. The control objective is to design a robust control law that ensures highaccuracy tracking performance. The tracking errors are defined as follows:

$$
\begin{aligned}
& e_{1}=x_{1}-x_{1 d} \\
& e_{2}=x_{2}-\dot{x}_{1 d}
\end{aligned}
$$

where $x_{1 d}$ and $\dot{x}_{1 d}$ are the desired angular position and velocity.

The sliding surface for the actual tracking error is constructed by

$$
s=e_{2}+\lambda e_{1}
$$

where $\lambda$ is a positive constant. To guarantee the convergence for the sliding variable (14), an auxiliary sliding surface is designed as

$$
\sigma=\dot{s}+k s
$$

where $k$ is a positive constant.

The time derivative of the auxiliary sliding surface $\sigma$ defined in (15) can be derived as

$$
\dot{\sigma}=\ddot{s}+k \dot{s}
$$


For the sake of simplicity, we use $f_{1}, \dot{f}_{1}, f_{2}$, and $g_{2}$ instead of $f_{1}(\mathbf{x}), \dot{f}_{1}(\mathbf{x}), f_{2}(\mathbf{x})$, and $g_{2}(\mathbf{x}, u)$. Taking the first and second-order derivatives of the sliding variable (14) and combining them with the system dynamics (11), one obtains

$$
\begin{aligned}
\dot{s} & =x_{3}+f_{1}+d_{1}-\ddot{x}_{1 d}+\lambda e_{2} \\
\ddot{s} & =f_{2}+g_{2} u+d_{2}+\dot{f}_{1}+\dot{d}_{1}-x_{1 d}^{(3)} \\
& +\lambda\left(x_{3}+f_{1}+d_{1}-\ddot{x}_{1 d}\right)
\end{aligned}
$$

where $x^{(3)}$ represents the third-order derivative of $x$.

Substituting (17) into (16), the derivative of $\sigma$ is given by

$$
\begin{aligned}
\dot{\sigma} & =f_{2}+d_{2}+g_{2} u+\dot{f}_{1}+\dot{d}_{1}-x_{1 d}^{(3)}+k \lambda\left(x_{2}-\dot{x}_{1 d}\right) \\
& +(\lambda+k)\left(x_{3}+f_{1}+d_{1}-\ddot{x}_{1 d}\right)
\end{aligned}
$$

Based on this, the equivalent control is synthesized as

$$
u_{e q}=\frac{1}{g_{2}}\left(\begin{array}{l}
-f_{2}-d_{2}-\dot{f}_{1}-\dot{d}_{1}+x_{1}^{(3 d)} \\
-(\lambda+k)\left(x_{3}+f_{1}+d_{1}-\ddot{x}_{1 d}\right) \\
-k \lambda\left(x_{2}-\dot{x}_{1 d}\right)
\end{array}\right)
$$

The switching control term is designed as

$$
u_{s w}=-\frac{1}{g_{2}}\left(\eta_{1} \operatorname{sgn}(\sigma)+\eta_{2} \sigma\right)
$$

where $\eta_{1}$ and $\eta_{2}$ are positive constants that are determined later.

Based on (19) and (20), the complete control law is derived as

$$
u=u_{e q}+u_{s w}
$$

Theorem 1. The control laws (19)-(21) guarantee the finite-time convergence of the auxiliary sliding variable without a singularity. Based on that, the tracking error asymptotically converges to zero in the case that the functions $f_{1}(\mathbf{x}), f_{2}(\mathbf{x})$, and $g_{2}(\mathbf{x}, u)$ are known and the angular velocity along with the lumped disturbances $d_{1}$ and $d_{2}$ are measurable.

Proof of Theorem 1. Consider the candidate Lyapunov function as

$$
V_{c}=\frac{1}{2} \sigma^{2}
$$

Taking the time derivative of (22), one obtains

$$
\dot{V}_{c}=\sigma \dot{\sigma}
$$

Substituting (19)-(21) into (23), we derive

$$
\dot{V}_{c} \leq-\eta_{1}|\sigma|
$$

Based on (23) and (24), one can obtain

$$
\dot{V}_{c} \leq-\eta \sqrt{2} V_{c}^{1 / 2}
$$

It can be observed from (25) that $V_{c}$ converges to zero in finite time with convergence time is computed as

$$
t_{r} \leq \frac{\sqrt{2}}{\eta} V_{c}^{1 / 2}(0)
$$


Based on this, it can be concluded that by using the control laws (19), (20), and (21), the auxiliary sliding variable converges to zero in finite time; hence, the control system is asymptotically stable.

This completes the proof of Theorem 1.

\subsection{Levant's High-Order Exact Differentiator}

To exactly compute the angular velocity and the angular acceleration of the inertial load, the first Levant's differentiator [29] is designed as

$$
\begin{aligned}
& \bar{x}_{1}=z_{1} ; \dot{z}_{1}=-\varsigma_{1}\left|z_{1}-x_{1}\right|^{3 / 4} \operatorname{sgn}\left(z_{1}-x_{1}\right)+z_{2} \\
& \bar{x}_{2}=z_{2} ; \dot{z}_{2}=-\varsigma_{2}\left|z_{2}-\dot{z}_{1}\right|^{2 / 3} \operatorname{sgn}\left(z_{2}-\dot{z}_{1}\right)+z_{3} \\
& \overline{\dot{x}}_{2}=z_{3} ; \dot{z}_{3}=-\varsigma_{3}\left|z_{3}-\dot{z}_{2}\right|^{1 / 2} \operatorname{sgn}\left(z_{3}-\dot{z}_{2}\right)+z_{4} \\
& \dot{z}_{4}=-\varsigma_{4} \operatorname{sgn}\left(z_{4}-\dot{z}_{3}\right)
\end{aligned}
$$

where $\varsigma_{1}, \varsigma_{2}, \varsigma_{4}$, and $\varsigma_{4}$ are positive constants and $\bar{x}_{1}, \bar{x}_{2}$, and $\overline{\dot{x}}_{2}$ denote the estimated position, velocity, and acceleration, respectively.

Similarly, the second Levant's differentiator to calculate the first-order derivative of load pressure is determined by

$$
\begin{aligned}
& \bar{x}_{3}=v_{1} ; \dot{v}_{1}=-\xi_{1}\left|v_{1}-x_{3}\right|^{2 / 3} \operatorname{sgn}\left(v_{1}-x_{3}\right)+v_{2} \\
& \dot{x}_{3}=v_{2} ; \dot{v}_{2}=-\xi_{2}\left|v_{2}-\dot{v}_{1}\right|^{1 / 2} \operatorname{sgn}\left(v_{2}-\dot{v}_{1}\right)+v_{3} \\
& \dot{v}_{3}=-\xi_{3} \operatorname{sgn}\left(v_{3}-\dot{v}_{2}\right)
\end{aligned}
$$

where $\xi_{1}, \xi_{2}$, and $\xi_{3}$ are positive constants; $\bar{x}_{3}$ and $\bar{x}_{3}$ denote estimates of load pressure and the first-order derivative, respectively.

Remark 1. The angular velocity and acceleration of the load and the derivative of load pressure of the HRA are computed based on the well-known Levant's exact differentiator [29] with negligible calculation error. The differentiation term of $(\bullet)$ is denoted by $(\boldsymbol{\bullet})$. For the sake of condensation, the dynamics of this differentiator are not presented in this paper.

\subsection{Unknown Dynamic Estimators}

Consider an arbitrary unknown smooth multivariate function $f(\mathbf{x})$ with $\mathbf{x}$ as the input vector. This function can be represented in RBF NN form as [39]

$$
f(\mathbf{x})=\mathbf{W}_{f}^{T} \mathbf{h}_{f}(\mathbf{x})+\varepsilon_{f}
$$

where $\mathbf{W}_{f}$ is the ideal neural network weights, $\mathbf{h}_{f}=\left[h_{j}\right]^{T}$ with $h_{j}$ represents the radialbasis function output of the $j$ th node in the hidden layer, and $\varepsilon_{f}$ denotes the function approximation error.

Assumption 4. The weight value and the approximation error of $N N$ are bounded as $\left\|\mathbf{W}_{f}\right\| \leq$ $W_{f M}$ and $\left|\varepsilon_{f}\right| \leq \varepsilon_{f M}$ where $W_{f M}$ and $\varepsilon_{f M}$ are positive constants.

The radial-basis function is usually chosen as the Gaussian function, which has the form [40]

$$
h_{j}=\exp \left(-\frac{\left\|\mathbf{x}-\mathbf{c}_{j}\right\|^{2}}{2 b_{j}^{2}}\right)
$$

where $\mathbf{c}_{j}$ is the center vector of the $j$ th node in the hidden layer, which has the same dimension as the input vector $\mathbf{x}$, and $\|\bullet\|$ represents the Euclid distance of a vector. 
The estimation of the function $f(\mathbf{x})$ is denoted by $\hat{f}(\mathbf{x})$, which is the output of RBF $\mathrm{NN}$ as

$$
\hat{f}(\mathbf{x})=\hat{\mathbf{W}}_{f}^{T} \mathbf{h}_{f}(\mathbf{x})
$$

In (11), since the system parameters are unknown, the functions $f_{1}, \dot{f}_{1}, f_{2}$, and $g_{2}$ are unknown smooth functions that are approximated by employing RBF NN-based approximators as

$$
\begin{aligned}
& f_{1}=\mathbf{W}_{f_{1}}^{T} \mathbf{h}_{f_{1}}(\mathbf{x})+\varepsilon_{f_{1}} \\
& \dot{f}_{1}=\mathbf{W}_{d f_{1}}^{T} \mathbf{h}_{d f_{1}}(\mathbf{x})+\varepsilon_{d f_{1}} \\
& f_{2}=\mathbf{W}_{f_{2}}^{T} \mathbf{h}_{f_{2}}(\mathbf{x})+\varepsilon_{f_{2}} \\
& g_{2}=\mathbf{W}_{g_{2}}^{T} \mathbf{h}_{g_{2}}(\boldsymbol{\varnothing})+\varepsilon_{g_{2}}
\end{aligned}
$$

where the weight values $\left\|\mathbf{W}_{f_{1}}\right\|,\left\|\mathbf{W}_{d f_{1}}\right\|,\left\|\mathbf{W}_{f_{2}}\right\|,\left\|\mathbf{W}_{g_{2}}\right\|$ and approximation errors $\varepsilon_{f_{1}}, \varepsilon_{d f_{1}}$, $\varepsilon_{f_{2}}, \varepsilon_{g_{2}}$ are bounded by $W_{f_{1} M}, W_{d f_{1} M}, W_{f_{2} M}, W_{g_{2} M}$ and $\varepsilon_{f_{1} M}, \varepsilon_{d f_{1} M}, \varepsilon_{f_{2} M}, \varepsilon_{g_{2} M}$, respectively; and the input vector $\boldsymbol{\varnothing}=\left[x_{1}, \bar{x}_{2}, x_{3}, u\right]^{T}$.

The estimates of $f_{1}, \dot{f}_{1}, f_{2}$, and $g_{2}$ are determined as

$$
\begin{aligned}
& \hat{f}_{1}=\hat{\mathbf{W}}_{f_{1}}^{T} \mathbf{h}_{f_{1}}(\mathbf{x}) \\
& \hat{f}_{1}=\hat{\mathbf{W}}_{d f_{1}}^{T} \mathbf{h}_{d f_{1}}(\mathbf{x}) \\
& \hat{f}_{2}=\hat{\mathbf{W}}_{f_{2}}^{T} \mathbf{h}_{f_{2}}(\mathbf{x}) \\
& \hat{g}_{2}=\hat{\mathbf{W}}_{g_{2}}^{T} \mathbf{h}_{g_{2}}(\boldsymbol{\varnothing})
\end{aligned}
$$

The approximation errors are determined by

$$
\begin{aligned}
& \tilde{f}_{1} \triangleq f_{1}-\hat{f}_{1}=\tilde{\mathbf{W}}_{f_{1}}^{T} \mathbf{h}_{f_{1}}+\varepsilon_{f_{1}} \\
& \tilde{f}_{1} \triangleq \dot{f}_{1}-\hat{f}_{1}=\tilde{\mathbf{W}}_{d f_{1}}^{T} \mathbf{h}_{d f_{1}}+\varepsilon_{d f_{1}} \\
& \tilde{f}_{2} \triangleq f_{2}-\hat{f}_{2}=\tilde{\mathbf{W}}_{f_{2}}^{T} \mathbf{h}_{f_{2}}+\varepsilon_{f_{2}} \\
& \tilde{g}_{2} \triangleq g_{2}-\hat{g}_{2}=\tilde{\mathbf{W}}_{g_{2}}^{T} \mathbf{h}_{g_{2}}+\varepsilon_{g_{2}}
\end{aligned}
$$

where

$$
\begin{aligned}
& \tilde{\mathbf{W}}_{f_{1}}=\mathbf{W}_{f_{1}}-\hat{\mathbf{W}}_{f_{1}} \\
& \tilde{\mathbf{W}}_{d f_{1}}=\mathbf{W}_{d f_{1}}-\hat{\mathbf{W}}_{d f_{1}} \\
& \tilde{\mathbf{W}}_{f_{2}}=\mathbf{W}_{f_{2}}-\hat{\mathbf{W}}_{f_{2}} \\
& \tilde{\mathbf{W}}_{g_{2}}=\mathbf{W}_{g_{2}}-\hat{\mathbf{W}}_{g_{2}}
\end{aligned}
$$

The adaption mechanisms are determined as

$$
\begin{aligned}
& \dot{\hat{\mathbf{W}}}_{f_{1}}=\gamma_{f_{1}}\left(\lambda+k-\frac{1}{\kappa_{1}}\right) \sigma \mathbf{h}_{f_{1}}-\Gamma_{f_{1}} \hat{\mathbf{W}}_{f_{1}} \\
& \dot{\hat{\mathbf{W}}}_{d f_{1}}=\gamma_{d f_{1}} \sigma \mathbf{h}_{d f_{1}}-\Gamma_{d f_{1}} \hat{\mathbf{W}}_{d f_{1}} \\
& \dot{\mathbf{W}}_{f_{2}}=\gamma_{f_{2}} \sigma \mathbf{h}_{f_{1}}-\Gamma_{f_{2}} \hat{\mathbf{W}}_{f_{2}} \\
& \dot{\hat{\mathbf{W}}}_{g_{2}}=\gamma_{g_{2}} \sigma u \mathbf{h}_{g_{2}}-\Gamma_{g_{2}} \hat{\mathbf{W}}_{g_{2}}
\end{aligned}
$$

where $\gamma_{f_{1}}, \gamma_{d f_{1}}, \gamma_{f_{2}}$, and $\gamma_{g_{2}}$ are learning rates of the neural networks, $\sigma$ is the auxiliary sliding variable defined in (15), $u$ is the control input, $\Gamma_{f_{1}}, \Gamma_{d f_{1}}, \Gamma_{f_{2}}$, and $\Gamma_{g_{2}}$ are positive constants, and $\kappa_{1}$ is the time constant of a disturbance observer, which is designed later. 


\subsection{Disturbance Observer Design}

To actively compensate for the effect of unmodeled disturbances, uncertain nonlinearities, external load, and the imperfection in approximating unknown continuous functions in system dynamics (11) on the control system, a pair of NN-based disturbance observers are proposed as

$$
\begin{aligned}
& \dot{\hat{d}}_{1}=\frac{1}{\kappa_{1}}\left(\overline{\dot{x}}_{2}-x_{3}-\hat{f}_{1}-\hat{d}_{1}\right) \\
& \dot{\hat{d}}_{2}=\frac{1}{\kappa_{2}}\left(\overline{\dot{x}}_{3}-\hat{f}_{2}-\hat{g}_{2} u-\hat{G}_{1}-\hat{d}_{2}\right)
\end{aligned}
$$

where $\kappa_{1}$ and $\kappa_{2}$ are small positive constants, $\hat{d}_{1}$ and $\hat{d}_{2}$ are estimates of $d_{1}$ and $d_{2}$, respectively. $\hat{G}_{1}$ is the NN-based approximation of $G_{1}=\tilde{g}_{2} u$, which is generated by the NN weighting approximation error of $g_{2}$.

The function $G_{1}$ is represented by $\mathrm{RBF} N \mathrm{~N}$ as

$$
G_{1}=\mathbf{W}_{G_{1}}^{T} \mathbf{h}_{G_{1}}(\boldsymbol{\varnothing})+\varepsilon_{G_{1}}
$$

where the weight value $\left\|\mathbf{W}_{G_{1}}\right\|$ and approximation error $\varepsilon_{G_{1}}$ are bounded by positive constants; i.e., $\left\|\mathbf{W}_{G_{1}}\right\| \leq W_{G_{1} M},\left|\varepsilon_{G_{1}}\right| \leq \varepsilon_{G_{1} M}$.

From (37), the approximated value $\hat{G}_{1}$ and the approximation error $\tilde{G}_{1}$ are given by

$$
\begin{aligned}
& \hat{G}_{1}=\hat{\mathbf{W}}_{G_{1}}^{T} \mathbf{h}_{G_{1}} \\
& \tilde{G}_{1}=\tilde{\mathbf{W}}_{G_{1}}^{T} \mathbf{h}_{G_{1}}+\varepsilon_{G_{1}}
\end{aligned}
$$

where $\tilde{\mathbf{W}}_{G_{1}}=\mathbf{W}_{G_{1}}-\hat{\mathbf{W}}_{G_{1}}$ and the weight is updated according to the following law:

$$
\dot{\hat{\mathbf{W}}}_{G_{1}}=\frac{1}{2} \gamma_{G_{1}} \sigma \mathbf{h}_{G_{1}}-\Gamma_{G_{1}} \hat{\mathbf{W}}_{G_{1}}
$$

where $\gamma_{G_{1}}$ and $\Gamma_{G_{1}}$ are positive constants. The disturbance estimation errors are defined by

$$
\begin{aligned}
& \tilde{d}_{1}=d_{1}-\hat{d}_{1} \\
& \tilde{d}_{2}=d_{2}-\hat{d}_{2}
\end{aligned}
$$

Assuming that the state derivatives are perfectly calculated, based on (11) and (37), the disturbance estimation error dynamics are obtained as

$$
\begin{aligned}
& \dot{\tilde{d}}_{1}=-\frac{1}{\kappa_{1}}\left(\tilde{f}_{1}+\tilde{d}_{1}\right)+\dot{d}_{1} \\
& \dot{\tilde{d}}_{2}=-\frac{1}{\kappa_{2}}\left(\tilde{f}_{2}+\tilde{G}_{1}+\tilde{d}_{2}\right)+\dot{d}_{2}
\end{aligned}
$$

Remark 2. With the help of the exact high-order differentiators (27) and (28), and when the system dynamics are assumed to be known-i.e., $\tilde{f}_{1}=0, \tilde{f}_{2}=0$, and $\tilde{g}_{2}=0$ - the disturbance observers (37) guarantee the uniformly asymptotic stability under the constant disturbances and boundedness stability under the time-varying disturbances. 
Based on (19)-(21), (27), (28), (33), and (37), the control laws are redesigned as

$$
\begin{aligned}
& u_{e q}=\frac{1}{\hat{g}_{2}}\left(\begin{array}{l}
-\hat{f}_{2}-\frac{1}{2} \hat{G}_{1}-\frac{1}{2} \hat{G}_{2}-\hat{d}_{2}-\hat{\hat{f}}_{1}+\dot{\hat{d}}_{1} \\
+x_{1 d}^{(3)}-(\lambda+k)\left(x_{3}+\hat{f}_{1}+\hat{d}_{1}-\ddot{x}_{1 d}\right) \\
-k \lambda\left(\bar{x}_{2}-\dot{x}_{1 d}\right)
\end{array}\right) \\
& u_{s w}=-\frac{1}{\hat{g}_{2}}\left[\eta_{1} \operatorname{sgn}(\sigma)+\eta_{2} \sigma\right] \\
& u=u_{e q}+u_{s w}
\end{aligned}
$$

where

$$
\begin{aligned}
& e_{1}=x_{1}-x_{1 d} ; e_{2}=\bar{x}_{2}-\dot{x}_{1 d} \\
& s=e_{2}+\lambda e_{1} ; \sigma=\ddot{s}+k \dot{s}
\end{aligned}
$$

and the function $G_{2}=\varepsilon_{g_{2}} u$ is approximated by adopting RBF NN as

$$
G_{2}=\mathbf{W}_{G_{2}}^{T} \mathbf{h}_{G_{2}}(\boldsymbol{\varnothing})+\varepsilon_{G_{2}}
$$

with $\left\|\mathbf{W}_{G_{2}}\right\| \leq W_{G_{2} M}$ and $\left|\varepsilon_{G_{2}}\right| \leq \varepsilon_{G_{2} M}$.

The approximation value of $G_{2}$ is determined by

$$
\hat{G}_{2}=\hat{\mathbf{W}}_{G_{2}}^{T} \mathbf{h}_{G_{2}}
$$

The weight update law is designed as

$$
\dot{\hat{\mathbf{W}}}_{G_{2}}=\frac{1}{2} \gamma_{G_{2}} \sigma \mathbf{h}_{G_{2}}-\Gamma_{G_{2}} \hat{\mathbf{W}}_{G_{2}}
$$

where $\gamma_{G_{2}}$ and $\Gamma_{G_{2}}$ are positive constants.

\section{Stability Analysis}

Theorem 2. For system (11), by using the control law (43) with unknown function approximators (33), (39) and (46), the pair of disturbance observers (37), adaptive mechanisms (36), (40), and (47), and differentiators (27) and (28), the ultimately uniformly bounded tracking performance is ensured in the presence of unknown dynamics and both mismatched and matched disturbances.

Proof of Theorem 2. Consider the candidate Lyapunov function as

$$
\begin{aligned}
V & =\frac{1}{2} \sigma^{2}+\frac{1}{2 \gamma_{f_{1}}} \tilde{\mathbf{W}}_{f_{1}}^{T} \tilde{\mathbf{W}}_{f_{1}}+\frac{1}{2 \gamma_{d f_{1}}} \tilde{\mathbf{W}}_{d f_{1}}^{T} \tilde{\mathbf{W}}_{d f_{1}}+\frac{1}{2 \gamma_{G_{1}}} \tilde{\mathbf{W}}_{G_{1}}^{T} \tilde{\mathbf{W}}_{G_{1}}+\frac{1}{2 \gamma_{G_{2}}} \tilde{\mathbf{W}}_{G_{2}}^{T} \tilde{\mathbf{W}}_{G_{2}} \\
& +\frac{1}{2 \gamma_{f_{2}}} \tilde{\mathbf{W}}_{f_{2}}^{T} \tilde{\mathbf{W}}_{f_{2}}+\frac{1}{2 \gamma_{g_{2}}} \tilde{\mathbf{W}}_{g_{2}}^{T} \tilde{\mathbf{W}}_{g_{2}}+\frac{1}{2} \tilde{d}_{1}^{2}+\frac{1}{2} \tilde{d}_{2}^{2}
\end{aligned}
$$

Taking the time derivative of (48), one obtains

$$
\begin{aligned}
\dot{V} & =\sigma \dot{\sigma}+\frac{1}{\gamma_{f_{1}}} \tilde{\mathbf{W}}_{f_{1}}^{T} \dot{\tilde{\mathbf{W}}}_{f_{1}}+\frac{1}{\gamma_{d f_{1}}} \tilde{\mathbf{W}}_{d f_{1}}^{T} \dot{\tilde{\mathbf{W}}}_{d f_{1}}+\frac{1}{\gamma_{G_{1}}} \tilde{\mathbf{W}}_{G_{1}}^{T} \dot{\tilde{\mathbf{W}}}_{G_{1}}+\frac{1}{\gamma_{G_{2}}} \tilde{\mathbf{W}}_{G_{2}}^{T} \dot{\tilde{\mathbf{W}}}_{G_{2}} \\
& +\frac{1}{\gamma_{f_{2}}} \tilde{\mathbf{W}}_{f_{2}}^{T} \dot{\tilde{\mathbf{W}}}_{f_{2}}+\frac{1}{\gamma_{g_{2}}} \tilde{\mathbf{W}}_{g_{2}}^{T} \dot{\tilde{\mathbf{W}}}_{g_{2}}+\tilde{d}_{1} \dot{\tilde{d}}_{1}+\tilde{d}_{2} \dot{\tilde{d}}_{2}
\end{aligned}
$$


Based on (18), (37), (42), and (43), (49) can be transformed into

$$
\begin{aligned}
\dot{V} & =\sigma\left(\begin{array}{l}
\tilde{f}_{2}+\frac{1}{2} \tilde{\mathbf{W}}_{g_{2}}^{T} \mathbf{h}_{g_{2}} u+\frac{1}{2} \tilde{G}_{1}+\frac{1}{2} \tilde{G}_{2}+\tilde{d}_{2} \\
+\tilde{f}_{1}+\dot{d}_{1}+\left(\lambda+k-\frac{1}{\kappa_{1}}\right)\left(\tilde{f}_{1}+\tilde{d}_{1}\right) \\
-\eta_{1} \operatorname{sgn}(\sigma)-\eta_{2} \sigma
\end{array}\right)-\frac{1}{\gamma_{f_{1}}} \tilde{\mathbf{W}}_{f_{1}}^{T} \dot{\hat{\mathbf{W}}}_{f_{1}}-\frac{1}{\gamma_{d f_{1}}} \tilde{\mathbf{W}}_{d f_{1}}^{T} \dot{\hat{\mathbf{W}}}_{d f_{1}} \\
& -\frac{1}{\gamma_{f_{2}}} \tilde{\mathbf{W}}_{f_{2}}^{T} \dot{\hat{\mathbf{W}}}_{f_{2}}-\frac{1}{\gamma_{g_{2}}} \tilde{\mathbf{W}}_{g_{2}}^{T} \dot{\hat{\mathbf{W}}}_{g_{2}}-\frac{1}{\gamma_{G_{1}}} \tilde{\mathbf{W}}_{G_{1}}^{T} \dot{\hat{\mathbf{W}}}_{G_{1}}-\frac{1}{\gamma_{G_{2}}} \tilde{\mathbf{W}}_{G_{2}}^{T} \dot{\mathbf{W}}_{G_{2}} \\
& +\tilde{d}_{1}\left(-\frac{1}{\kappa_{1}}\left(\tilde{f}_{1}+\tilde{d}_{1}\right)+\dot{d}_{1}\right)+\tilde{d}_{2}\left(-\frac{1}{\kappa_{2}}\left(\tilde{f}_{2}+\tilde{G}_{1}+\tilde{d}_{2}\right)+\dot{d}_{2}\right)
\end{aligned}
$$

Applying adaptation mechanisms (33) and (40), (47) and (50) become

$$
\begin{aligned}
\dot{V} & =\frac{\Gamma_{f_{1}}}{\gamma_{f_{1}}} \tilde{\mathbf{W}}_{f_{1}}^{T} \hat{\mathbf{W}}_{f_{1}}+\frac{\Gamma_{d f_{1}}}{\gamma_{d f_{1}}} \tilde{\mathbf{W}}_{d f_{1}}^{T} \hat{\mathbf{W}}_{d f_{1}}+\frac{\Gamma_{f_{2}}}{\gamma_{f_{2}}} \tilde{\mathbf{W}}_{f 2}^{T} \hat{\mathbf{W}}_{f_{2}}+\frac{\Gamma_{g 2}}{\gamma_{g_{2}}} \tilde{\mathbf{W}}_{g 2}^{T} \hat{\mathbf{W}}_{g_{2}}+\frac{\Gamma_{G_{1}}}{\gamma_{G_{1}}} \tilde{\mathbf{W}}_{G_{1}}^{T} \hat{\mathbf{W}}_{G_{1}} \\
& +\frac{\Gamma_{G_{2}}}{\gamma_{G_{2}}} \tilde{\mathbf{W}}_{G_{2}}^{T} \hat{\mathbf{W}}_{G_{2}}+\left(\lambda+k-\frac{1}{\kappa_{1}}\right) \sigma \varepsilon_{f_{1}}+\sigma \varepsilon_{d f_{1}}+\sigma \varepsilon_{f_{2}}+\frac{1}{2} \sigma \varepsilon_{G_{1}}+\frac{1}{2} \sigma \varepsilon_{G_{2}} \\
& +\left(\lambda+k-\frac{1}{\kappa_{1}}\right) \sigma \tilde{d}_{1}+\sigma \dot{d}_{1}+\sigma \tilde{d}_{2}-\eta_{1}|\sigma|-\eta_{2} \sigma^{2}-\frac{1}{\kappa_{1}} \tilde{d}_{1} \tilde{\mathbf{W}}_{f_{1}}^{T} \mathbf{h}_{f_{1}}-\frac{1}{\kappa_{1}} \tilde{d}_{1} \varepsilon_{f_{1}} \\
& -\frac{1}{\kappa_{1}} \tilde{d}_{1}^{2}+\tilde{d}_{1} \dot{d}_{1}-\frac{1}{\kappa_{2}} \tilde{d}_{2} \tilde{\mathbf{W}}_{f_{2}}^{T} \mathbf{h}_{f_{2}}-\frac{1}{\kappa_{2}} \tilde{d}_{2} \varepsilon_{f_{2}}-\frac{1}{\kappa_{2}} \tilde{d}_{2} \tilde{\mathbf{W}}_{G_{1}}^{T} \mathbf{h}_{G_{1}}-\frac{1}{\kappa_{2}} \tilde{d}_{2} \varepsilon_{G}-\frac{1}{\kappa_{2}} \tilde{d}_{2}^{2}+\tilde{d}_{2} \dot{d}_{2}
\end{aligned}
$$

Based on the Cauchy-Schwarz inequality, the following inequality holds:

$$
\left(\mathbf{W}^{\mathrm{T}} \mathbf{h}\right)^{2} \leq\left(\mathbf{W}^{\mathrm{T}} \mathbf{W}\right)\left(\mathbf{h}^{\mathrm{T}} \mathbf{h}\right)
$$

Applying Young's inequality and (52), (51) can be transformed into

$$
\begin{aligned}
\dot{V} & \leq-\left(\eta_{2}-\frac{5 \lambda}{2}-\frac{5 k}{2}+\frac{5}{2 \kappa_{1}}-4\right) \sigma^{2}-\left(\frac{\Gamma_{f_{1}}}{2 \gamma_{f_{1}}}-\frac{2 \lambda_{f_{1}}}{\kappa_{1}}\right) \tilde{\mathbf{W}}_{f_{1}}^{T} \tilde{\mathbf{W}}_{f_{1}}-\frac{\Gamma_{d f_{1}}}{2 \gamma_{d f_{1}}} \tilde{\mathbf{W}}_{d f_{1}}^{T} \tilde{\mathbf{W}}_{d f_{1}} \\
& -\left(\frac{\Gamma_{f_{2}}}{2 \gamma_{f_{2}}}-\frac{2 \lambda_{f_{2}}}{\kappa_{2}}\right) \tilde{\mathbf{W}}_{f 2}^{T} \tilde{\mathbf{W}}_{f_{2}}-\frac{\Gamma_{g 2}}{2 \gamma_{g_{2}}} \tilde{\mathbf{W}}_{g 2}^{T} \tilde{\mathbf{W}}_{g_{2}}-\left(\frac{\Gamma_{G_{1}}}{2 \gamma_{G_{1}}}-\frac{2 \lambda_{G_{1}}}{\kappa_{2}}\right) \tilde{\mathbf{W}}_{G_{1}}^{T} \tilde{\mathbf{W}}_{G_{1}} \\
& -\frac{\Gamma_{G_{2}}}{2 \gamma_{G_{2}}} \tilde{\mathbf{W}}_{G_{2}}^{T} \tilde{\mathbf{W}}_{G_{2}}-\left(\frac{7}{8 \kappa_{1}}-\frac{\lambda}{8}-\frac{k}{8}-\frac{1}{2}\right) \tilde{d}_{1}^{2}-\left(\frac{1}{2 \kappa_{2}}-\frac{5}{8}\right) \tilde{d}_{2}^{2}+\frac{\Gamma_{f_{1}}}{2 \gamma_{f_{1}}} W_{f_{1} M}^{2} \\
& +\frac{\Gamma_{d f_{1}}}{2 \gamma_{d f_{1}}} W_{d f_{1} M}^{2}+\frac{\Gamma_{f_{2}}}{2 \gamma_{f_{2}}} W_{f_{2} M}^{2}+\frac{\Gamma_{g 2}}{2 \gamma_{g_{2}}} W_{g_{2} M}^{2}+\frac{\Gamma_{G_{1}}}{2 \gamma_{G_{1}}} W_{G_{1} M}^{2}+\frac{\Gamma_{G_{2}}}{2 \gamma_{G_{2}}} W_{G_{2} M}^{2} \\
& +\left(\frac{\lambda}{2}+\frac{k}{2}+\frac{3}{2 \kappa_{1}}\right) \varepsilon_{f_{1} M}^{2}+\frac{\varepsilon_{d f_{1} M}^{2}}{2}+\left(\frac{1}{2}+\frac{2}{\kappa_{2}}\right) \varepsilon_{f_{2} M}^{2}+\left(\frac{1}{4}+\frac{2}{\kappa_{2}}\right) \varepsilon_{G_{1} M}^{2} \\
& +\frac{1}{4} \varepsilon_{G_{2} M}^{2}+\delta_{1}^{2}+\frac{\delta_{2}^{2}}{2}
\end{aligned}
$$

where $\lambda_{f_{1}}, \lambda_{f_{2}}$, and $\lambda_{G_{1}}$ are the number of nodes in the hidden layers of NNs for approximating $f_{1}, f_{2}$, and $G_{1}$, respectively; control gains, disturbance observer parameters, and NN parameters are chosen such that 


$$
\begin{aligned}
& \lambda+k-\frac{1}{\kappa_{1}}>0 ; \eta_{2}-\frac{5 \lambda}{2}-\frac{5 k}{2}+\frac{5}{2 \kappa_{1}}-4>0 \\
& \frac{\Gamma_{f_{1}}}{2 \gamma_{f_{1}}}-\frac{2 \lambda_{f_{1}}}{\kappa_{1}}>0 ; \frac{\Gamma_{f_{2}}}{2 \gamma_{f_{2}}}-\frac{2 \lambda_{f_{2}}}{\kappa_{2}}>0 ; \frac{\Gamma_{G_{1}}}{2 \gamma_{G_{1}}}-\frac{2 \lambda_{G_{1}}}{\kappa_{2}}>0 \\
& \frac{7}{8 \kappa_{1}}-\frac{\lambda}{8}-\frac{k}{8}-\frac{1}{2}>0 ; \frac{1}{2 \kappa_{2}}-\frac{5}{8}>0
\end{aligned}
$$

Equation (53) can be rewritten in a simple form as

$$
\dot{V} \leq-\Pi V+\psi
$$

where

$$
\Pi=\min \left\{\begin{array}{l}
2 \eta_{2}-5 \lambda-5 k+\frac{5}{\kappa_{1}}-8 ; \frac{\Gamma_{f_{1}}}{\gamma_{f_{1}}}-\frac{4 \lambda_{f_{1}}}{\kappa_{1}} ; \frac{\Gamma_{d f_{1}}}{\gamma_{d f_{1}}} ; \frac{\Gamma_{f_{2}}}{\gamma_{f_{2}}}-\frac{4 \lambda_{f_{2}}}{\kappa_{2}} ; \frac{\Gamma_{g^{2}}}{\gamma_{g_{2}}} ; \\
\frac{\Gamma_{G_{1}}}{\gamma_{G_{1}}}-\frac{4 \lambda_{G_{1}}}{\kappa_{2}} ; \frac{\Gamma_{G_{2}}}{\gamma_{G_{2}}} ; \frac{7}{4 \kappa_{1}}-\frac{\lambda}{4}-\frac{k}{4}-1 ; \frac{1}{\kappa_{2}}-\frac{5}{4}
\end{array}\right\}
$$

and

$$
\begin{aligned}
\psi & =\frac{\Gamma_{f_{1}}}{2 \gamma_{f_{1}}} W_{f_{1} M}^{2}+\frac{\Gamma_{d f_{1}}}{2 \gamma_{d f_{1}}} W_{d f_{1} M}^{2}+\frac{\Gamma_{f_{2}}}{2 \gamma_{f_{2}}} W_{f_{2} M}^{2} \\
& +\frac{\Gamma_{g_{2}}}{2 \gamma_{g_{2}}} W_{g_{2} M}^{2}+\frac{\Gamma_{G_{1}}}{2 \gamma_{G_{1}}} W_{G_{1} M}^{2}+\frac{\Gamma_{G_{2}}}{2 \gamma_{G_{2}}} W_{G_{2} M}^{2} \\
& +\left(\frac{\lambda}{2}+\frac{k}{2}+\frac{7}{2 \kappa_{1}}\right) \varepsilon_{f_{1} M}^{2}+\frac{\varepsilon_{d f_{1} M}^{2}}{2}+\left(\frac{1}{2}+\frac{2}{\kappa_{2}}\right) \varepsilon_{f_{2} M}^{2} \\
& +\left(\frac{1}{4}+\frac{2}{\kappa_{2}}\right) \varepsilon_{G_{1} M}^{2}+\frac{1}{4} \varepsilon_{G_{2} M}^{2}+\delta_{1}^{2}+\frac{\delta_{2}^{2}}{2}
\end{aligned}
$$

From (55), one obtains

$$
V \leq V(0) e^{-\Pi t}+\frac{\psi}{\Pi}\left(1-e^{-\Pi t}\right)
$$

Based on this, one can conclude that $\sigma$ is bounded, which implies that the tracking errors of the system are also bounded. Therefore, the ultimately uniformly bounded tracking performance of the system is guaranteed by using the proposed control strategy.

Hence, Theorem 2 is completely proven.

Remark 3. Firstly, the parameters $\kappa_{1}$ and $\kappa_{2}$ of disturbance observers (37) are chosen to be as small as possible based on the sampling time to achieve high estimation accuracy; however, the selection of the excessively small disturbance observer gains may make the disturbance observers unstable. Consequently, the remaining parameters, including controller gains in (14) and (15), parameters of adaptation mechanisms (36), (40) and (47) of RBF-NNs, are designed based on the closed-loop system stability condition (54).

\section{Numerical Simulation}

\subsection{Simulation Setup}

To verify the effectiveness of the proposed control strategy, the hydraulic servo system [38] is adopted. System parameters for the simulation are given in Table 1. 
Table 1. System parameters of the studied EHSS.

\begin{tabular}{|c|c|c|c|c|c|}
\hline Parameter & Unit & Value & Parameter & Unit & Value \\
\hline$J$ & $\mathrm{~kg} \cdot \mathrm{m}^{2}$ & 0.2 & $C_{0}$ & $\mathrm{~m}^{3} \cdot \mathrm{s}^{-1} \cdot \mathrm{Pa}^{-2}$ & 0 \\
\hline$B$ & $\mathrm{~N} \cdot \mathrm{m} \cdot \mathrm{s} \cdot \mathrm{rad}^{-1}$ & 90 & $C_{1}$ & $\mathrm{~m}^{3} \cdot \mathrm{s}^{-1} \cdot \mathrm{Pa}^{-1}$ & $1 \times 10^{-12}$ \\
\hline$D_{m}$ & $\mathrm{~m}^{3} \cdot \mathrm{rad}^{-1}$ & $5.8 \times 10^{-5}$ & $C_{2}$ & $\mathrm{~m}^{3} \cdot \mathrm{s}^{-1}$ & 0 \\
\hline$\beta_{e}$ & Pa or $\mathrm{N} \cdot \mathrm{m}^{-2}$ & $7 \times 10^{8}$ & $P_{S}$ & $\mathrm{~Pa}$ & $1 \times 10^{7}$ \\
\hline$k_{t}$ & $\mathrm{~m}^{3} \cdot \mathrm{s}^{-1} \cdot \mathrm{V}^{-1} \cdot \mathrm{Pa}^{-1 / 2}$ & $1.1969 \times 10^{-8}$ & $V_{t}$ & $\mathrm{~m}^{3}$ & $1.16 \times 10^{-4}$ \\
\hline$\tau_{\mathrm{C} 0}$ & $\mathrm{~N} \cdot \mathrm{m} \cdot \mathrm{s} \cdot \mathrm{rad}^{-1}$ & 35 & $\omega_{s}$ & $\mathrm{rad} \cdot \mathrm{s}^{-1}$ & 0.01 \\
\hline$\tau_{S 0}$ & $\mathrm{~N} \cdot \mathrm{m} \cdot \mathrm{s} \cdot \mathrm{rad}^{-1}$ & 40 & & & \\
\hline
\end{tabular}

To illustrate the superiority of the proposed method, some controllers are employed for comparison as follows:

1. The proposed controller with the control parameters $\lambda=1000, k=150, \eta_{1}=1000$, and $\eta_{2}=4.1 \times 10^{4}$. The parameters for the first Levant's differentiator are chosen as $\varsigma_{1}=100, \varsigma_{2}=50, \varsigma_{3}=100$, and $\varsigma_{4}=100$. For the second differentiator $\xi_{1}=350, \xi_{2}=1.5 \times 10^{4}$, and $\xi_{3}=1.5 \times 10^{4}$ are selected. The RBF NNs are designed with 51 nodes in the hidden layer, and their parameters are $\gamma_{f_{1}}=0.15, \gamma_{d f_{1}}=0.2$, $\gamma_{f_{2}}=0.02, \gamma_{g_{2}}=0.1, \gamma_{G_{1}}=0.01, \gamma_{G_{2}}=0.1, \Gamma_{f_{1}}=10^{3}, \Gamma_{d f_{1}}=10^{-7}, \Gamma_{f_{2}}=10^{3}$, $\Gamma_{g_{2}}=10^{-7}, \Gamma_{G_{1}}=2 \times 10^{3}$, and $\Gamma_{G_{2}}=10^{-7}$. The disturbance observer gains are chosen as $\kappa_{1}=0.005$ and $\kappa_{2}=0.005$.

2. RBF NN-based sliding mode control without disturbance observer (RBF-SMC), in which larger controller gains are designed $\eta_{1}=1500$ and $\eta_{2}=4.1 \times 10^{4}$ to attenuate the effects of disturbances while the avoidance of the chattering phenomenon is guaranteed. The structures and parameters of RBF NNs are the same as the proposed method.

3. Extended state observer-based backstepping controller (ESO-BC) [27], with the assumption that nominal system parameters are known.

The control law is designed as

$$
\begin{aligned}
& u=\frac{1}{g_{20}}\left(-f_{20}-\hat{d}_{2}+\dot{\alpha}_{2}-k_{3}\left(x_{3}-\alpha_{2}\right)\right) \\
& \alpha_{2}=-f_{10}+\dot{\alpha}_{1}-k_{2} z_{2}-\hat{d}_{1} \\
& \alpha_{1}=\dot{x}_{1 d}-k_{1}\left(x_{1}-x_{1 d}\right)
\end{aligned}
$$

where $z_{1}=x_{1}-x_{1 d}, z_{2}=\hat{x}_{2}-\alpha_{1}, z_{3}=x_{3}-\alpha_{2}$, and the nominal dynamic functions are

$$
\begin{aligned}
& f_{10}=-\frac{1}{J} B_{0} \hat{x}_{2}-\left(\tau_{c 0}+\tau_{s 0} e^{\left(-\frac{\left|\hat{x}_{2}\right|}{\omega_{s}}\right)}\right) \tanh \left(c_{f} \hat{x}_{2}\right) \\
& f_{20}=-\frac{4 D_{m}^{2} \beta_{e 0}}{J V_{t 0}} \hat{x}_{2}-\frac{4 \beta_{e 0} J C_{20}}{V_{t 0} D_{m}} x_{3} \\
& g_{20}=\frac{4 \beta_{e 0} k_{t 0}}{V_{t 0}} \sqrt{P_{s}-\operatorname{sign}(u) \frac{J}{D_{m}} x_{3}}
\end{aligned}
$$

The two ESOs are designed as

$$
\begin{gathered}
\left\{\begin{array}{l}
\dot{\hat{x}}_{1}=\hat{x}_{2}+3 \omega_{e 1}\left(x_{1}-\hat{x}_{1}\right) \\
\hat{x}_{2}=x_{3}+f_{10}\left(\hat{x}_{2}\right)+\hat{d}_{1}+3 \omega_{1}^{2}\left(x_{1}-\hat{x}_{1}\right) \\
\dot{\hat{d}}_{1}=\omega_{1}^{3}\left(x_{1}-\hat{x}_{1}\right)
\end{array}\right. \\
\left\{\begin{array}{l}
\dot{\hat{x}}_{3}= \\
f_{20}\left(\hat{x}_{2}, x_{3}\right)+g_{20}\left(x_{3}, u\right) u+2 \omega_{2}\left(x_{3}-\hat{x}_{3}\right) \\
\dot{\hat{d}}_{2}=\omega_{2}^{2}\left(x_{3}-\hat{x}_{3}\right)
\end{array}\right.
\end{gathered}
$$


where $f_{10}\left(x_{2}\right), f_{20}\left(x_{2}, x_{3}\right)$, and $g_{20}\left(x_{3}, u\right)$ are nominal system dynamic functions using known nominal system parameters, $\omega_{1}$ and $\omega_{2}$ are the observer gains, $\hat{d}_{1}$ and $\hat{d}_{2}$ are the estimates of mismatched and matched disturbances, respectively, and $\hat{x}_{i}$ is the estimated value of the system state $x_{i}$. The control parameters and observer gains are chosen as $k_{1}=450, k_{2}=350$, and $k_{3}=350 ; \omega_{1}=1000$ and $\omega_{2}=1000$, respectively, to make the tracking performance as good as possible without chattering issues. In this case, the nominal system parameters, which are slightly different from the actual system parameters, are employed to design the backstepping control law, as illustrated in Table 2.

4. Proportional-Integral-Derivative (PID) controller with controller gains manually tuned to make the tracking performance as good as possible without chattering in the control input as $K_{P}=750, K_{I}=500$, and $K_{D}=0.02$.

Table 2. Nominal system parameters of the EHSS for backstepping control design.

\begin{tabular}{cccccc}
\hline Parameter & Unit & Value & Parameter & Unit & Value \\
\hline$B_{0}$ & $\mathrm{~N} \cdot \mathrm{m} \cdot \mathrm{s} \cdot \mathrm{rad}^{-1}$ & 80 & $C_{10}$ & $\mathrm{~m}^{3} \cdot \mathrm{s}^{-1} \cdot \mathrm{Pa}^{-1}$ & $1 \times 10^{-12}$ \\
$\beta_{e 0}$ & $\mathrm{~Pa}$ or N $\cdot \mathrm{m}^{-2}$ & $7 \times 10^{8}$ & $C_{20}$ & $\mathrm{~m}^{3} \cdot \mathrm{s}^{-1}$ & 0 \\
$C_{00}$ & $\mathrm{~m}^{3} \cdot \mathrm{s}^{-1} \cdot \mathrm{Pa}^{-2}$ & 0 & $V_{t 0}$ & $\mathrm{~m}^{3}$ & $1 \times 10^{-4}$ \\
$k_{t 0}$ & $\mathrm{~m}^{3} \cdot \mathrm{s}^{-1} \cdot \mathrm{V}^{-1} \cdot \mathrm{Pa}^{-1 / 2}$ & $1 \times 10^{-8}$ & & & \\
\hline
\end{tabular}

The time-varying sinusoidal-like mismatched and matched disturbances $d_{1}(t)$ and $d_{2}(t)$ are intentionally injected into the control system for performance examination as follows:

$$
\begin{array}{ll}
d_{1}(t)=1.5 \times 10^{3} \sin (\pi t) & \left(\mathrm{rad} \cdot \mathrm{s}^{-2}\right) \\
d_{2}(t)=10^{6} \sin (\pi t) & \left(\mathrm{rad} \cdot \mathrm{s}^{-3}\right)
\end{array}
$$

where the magnitudes of these disturbances are chosen based on the specifications of the EHSS.

Performance indexes-i.e., the maximum, average, and standard deviation of the tracking errors [38] —are used to measure the efficiency of the aforementioned controllers. These criteria are defined as follows:

(i) Maximal absolute tracking error:

$$
M_{e}=\max _{i=1, \ldots, N}\left\{\left|e_{1}(i)\right|\right\}
$$

(ii) Average tracking error:

$$
\mu_{e}=\frac{1}{N} \sum_{i=1}^{N}\left|e_{1}(i)\right|
$$

(iii) Standard deviation of the tracking errors:

$$
\sigma_{e}=\sqrt{\frac{1}{N} \sum_{i=1}^{N}\left(\left|e_{1}(i)\right|-\mu_{e}\right)^{2}}
$$

\subsection{Simulation Results}

\subsubsection{Slow-Motion Reference Trajectory}

In this case, the smooth reference trajectory is chosen to evaluate the effectiveness of the proposed controller as

$$
x_{1 d}(t)=\frac{\pi}{8}\left(1-\cos \left(\frac{\pi}{2} t\right)\right)\left(1-e^{-t}\right)(\mathrm{rad})
$$


The tracking performances and tracking errors of all four controllers are depicted in Figures 3 and 4, respectively. As shown in Figure 3, all four controllers guarantee that the system output tracks the desired trajectory at an acceptable level. Nevertheless, from Figure 4, it is obviously seen that the proposed controller performs better than others with the smallest tracking errors, whereas the ESO-BC controller can track the reference trajectory more closely than RBF-SMC and PID controllers. For more details, the final tracking accuracies of all controllers are indicated via the three performance indexes given in Table 3. It is clear that, although it has some robustness, the PID controller can do little with uncertainties and disturbances due to the lack of model-based compensation mechanisms. Consequently, it exhibits the worst tracking performance in all performance indexes (maximal value, average, and standard deviation of the tracking error are about 0.4684 degrees, 0.2169 degrees, and 0.1326 degrees, respectively). Meanwhile, with the support of ESOs (60), both mismatched and matched disturbances are estimated and efficiently compensated; based on that, the ESO-BC controller (maximal error about 0.0315 degrees) achieves better tracking performance in comparison with the RBF-SMC controller (maximal error about 0.0358 degrees). It is noted that the maximal tracking error is only about 0.0148 degrees in the case of the proposed controller. The results demonstrate the effectiveness of the suggested control strategy in which the combination of DOBs and NNs is able to effectively deal with full unknown system dynamics and both mismatched and matched disturbances.

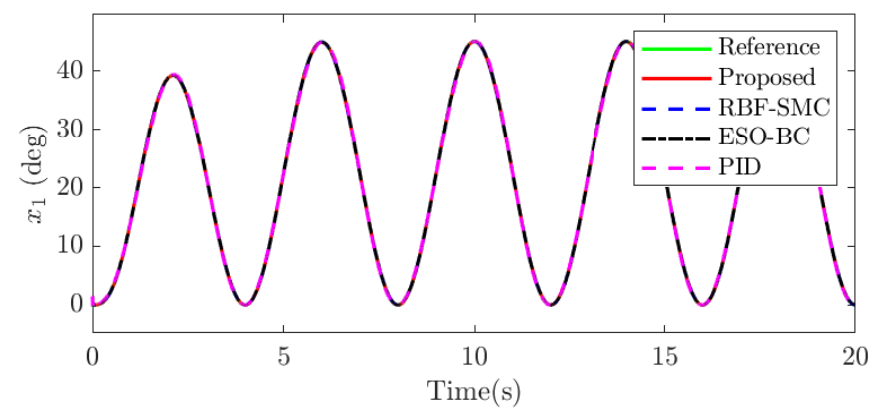

Figure 3. The tracking performance of the proposed strategy compared with other control laws with slow-motion reference trajectory.

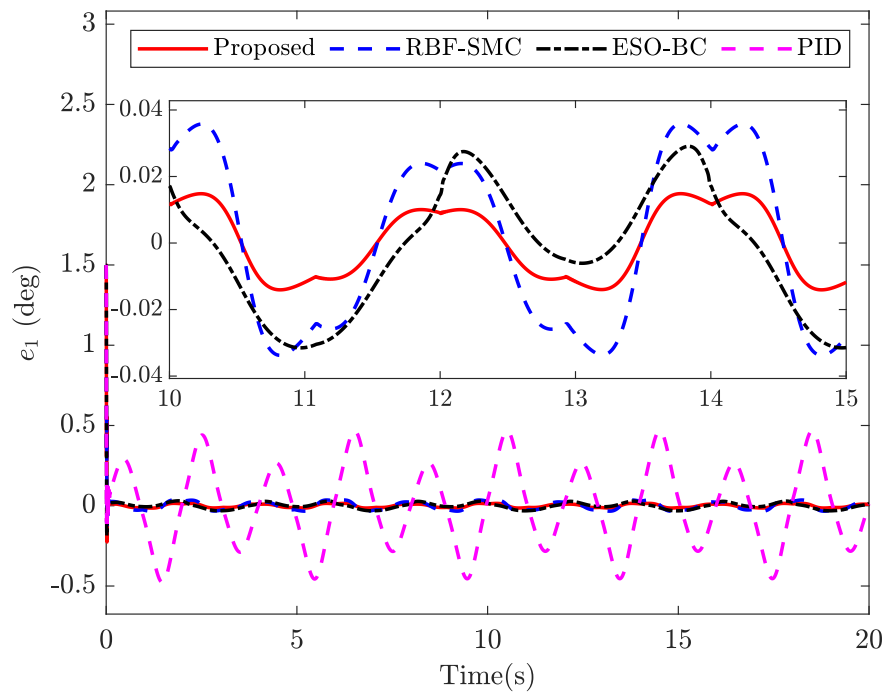

Figure 4. The tracking errors of the considered four control strategies with slow-motion reference trajectory. 
Table 3. Performance indexes in slow-motion reference trajectory of all considered controllers

\begin{tabular}{cccc}
\hline Controller & $\boldsymbol{M}_{\boldsymbol{e}}$ (Degree) & $\mu_{\boldsymbol{e}}$ (Degree) & $\sigma_{\boldsymbol{e}}$ (Degree) \\
\hline Proposed Controller & 0.0148 & 0.0097 & 0.0038 \\
RBF-SMC Controller & 0.0358 & 0.0233 & 0.0092 \\
ESO-BC Controller & 0.0315 & 0.0151 & 0.0104 \\
PID Controller & 0.4684 & 0.2169 & 0.1326 \\
\hline
\end{tabular}
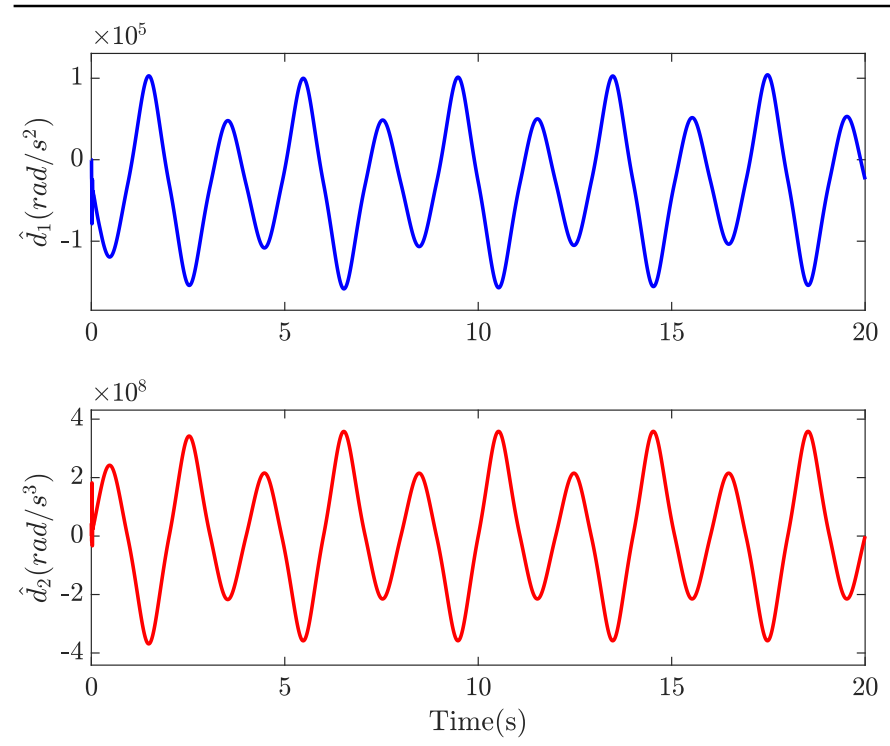

Figure 5. The estimates of the mismatched and matched disturbances of the proposed method.
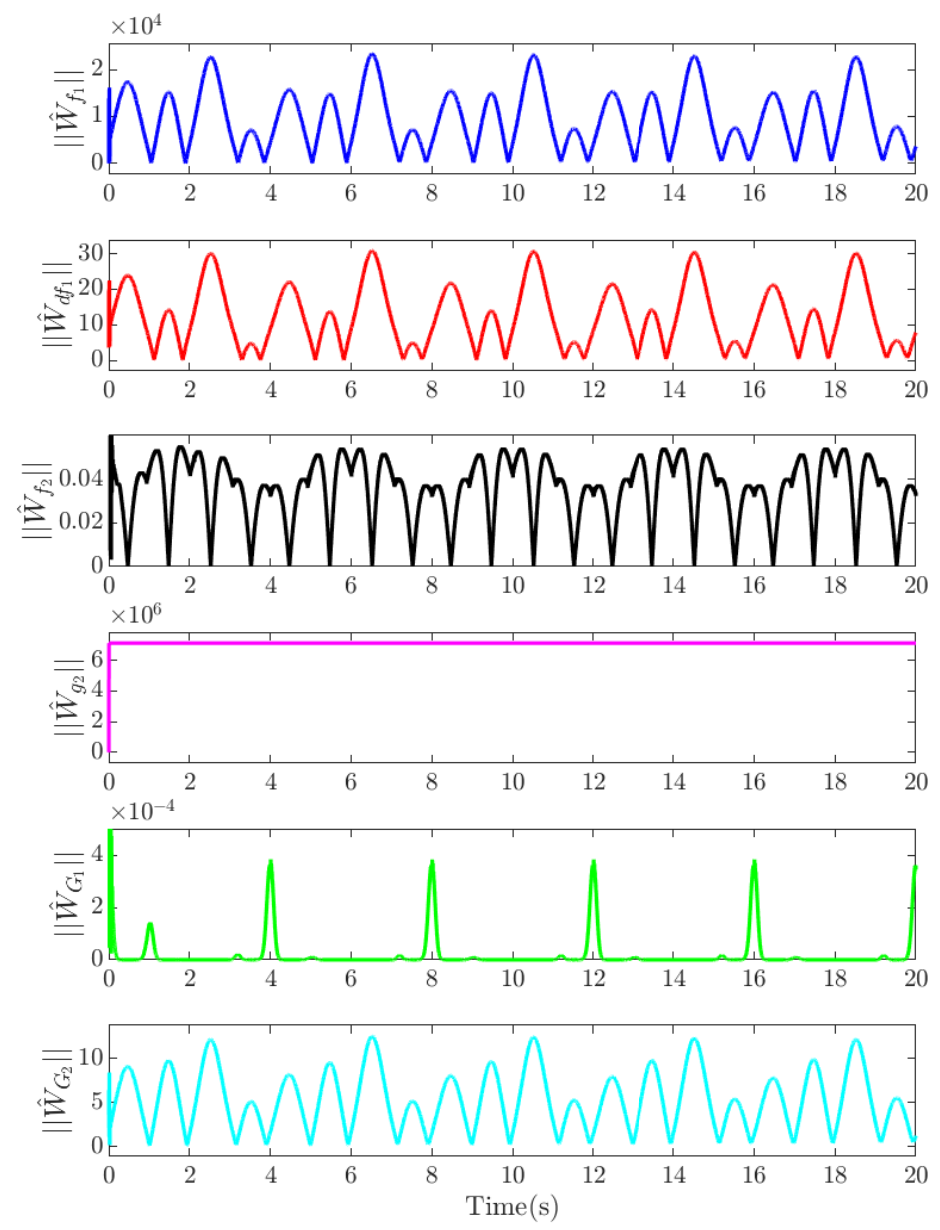

Figure 6. The weighting values of RFB NNs employed of the proposed method. 
The estimates of the mismatched and matched grouped disturbances and the weighting values of RBF NNs for approximating all unknown dynamic functions are illustrated in Figures 5 and 6, respectively. It is noted that from Figure 6 that the weighting values of RFB NNs are smooth and bounded. Moreover, the estimates of both mismatched and matched disturbances have the same frequencies as the injected disturbances (61). However, they are not in sinusoidal shapes, since the estimates of disturbances also include the approximation errors of RBF NNs. The control inputs of all controllers are depicted in Figure 7 . With the same desired reference trajectory, the control input shapes of all controllers are relatively similar. The interesting thing is that the control input is generated without chattering by the proposed controller, which demonstrates the effectiveness of the combination between RBF NNs and DOBs in dealing with the problem of full model uncertainties and disturbance of the recommended control method.

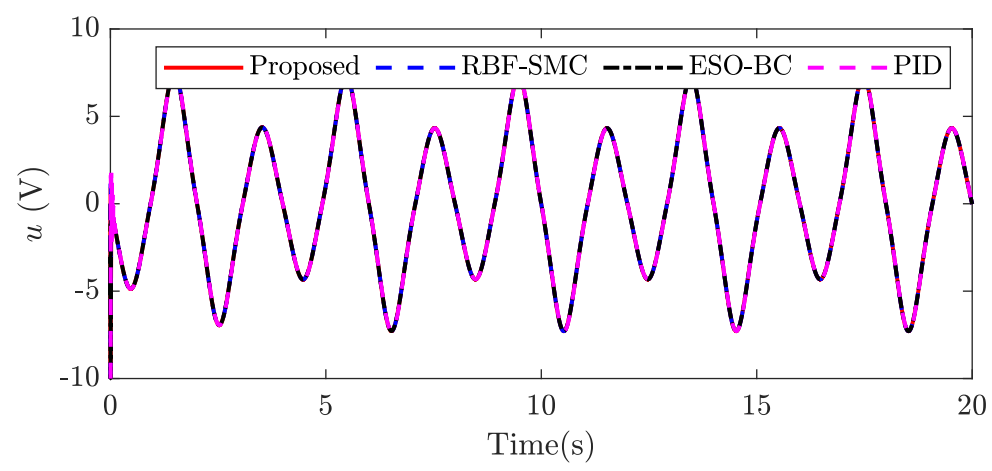

Figure 7. The control signals of all controllers with slow-motion reference trajectory.

\subsubsection{Fast-Motion Reference Trajectory}

For further capability investigation of the suggested control algorithm, a smooth fast-motion reference trajectory is chosen as

$$
x_{1 d}(t)=\frac{\pi}{16}(1-\cos (2 \pi t))\left(1-e^{-t}\right)(\operatorname{rad})
$$

The tracking characteristics of all controllers with the fast-motion reference trajectory are illustrated in Figure 8. As in the above case, all controllers still follow the desired trajectory. Due to the bandwidth limitations of EHSSs, the tracking errors of all control approaches depicted in Figure 9 rise remarkably along with the increase in the frequency of the reference trajectory. The tracking accuracies of all controllers in terms of the three performance indexes are illustrated in Table 4. As shown, the tracking performances of the PID controller and the RBF-SMC controller significantly degrade with the maximal final tracking errors, which are 0.5364 degrees and 0.0621 degrees, respectively. Meanwhile, the ESO-BC controller demonstrates robustness against uncertainties and disturbances based on the support of ESOs and known nominal system dynamics. However, it is worth noting that the proposed controller tracks the reference trajectory more accurately than others. This once again demonstrates the effectiveness of the recommended method in dealing with full model uncertainties and disturbances. 


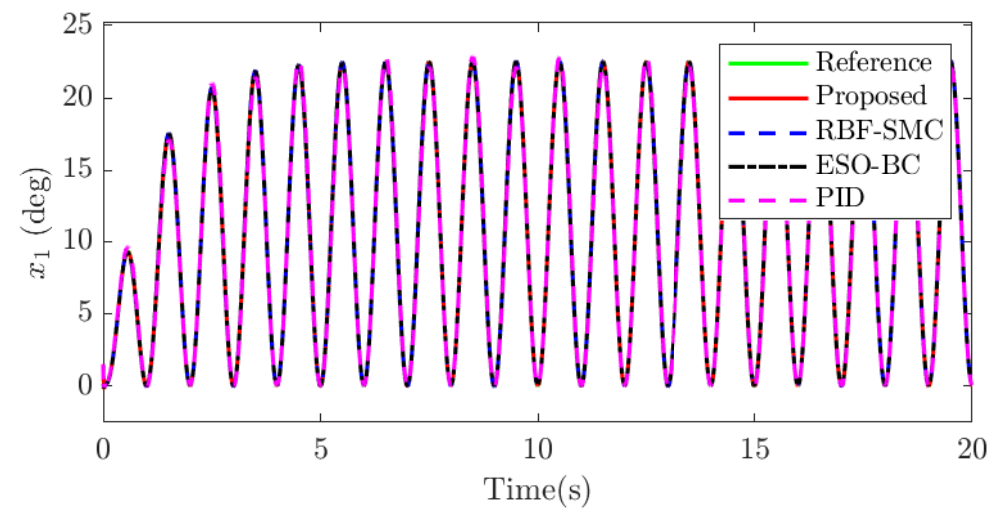

Figure 8. The tracking performance of the proposed strategy compared with other control laws in fast-motion reference trajectory.

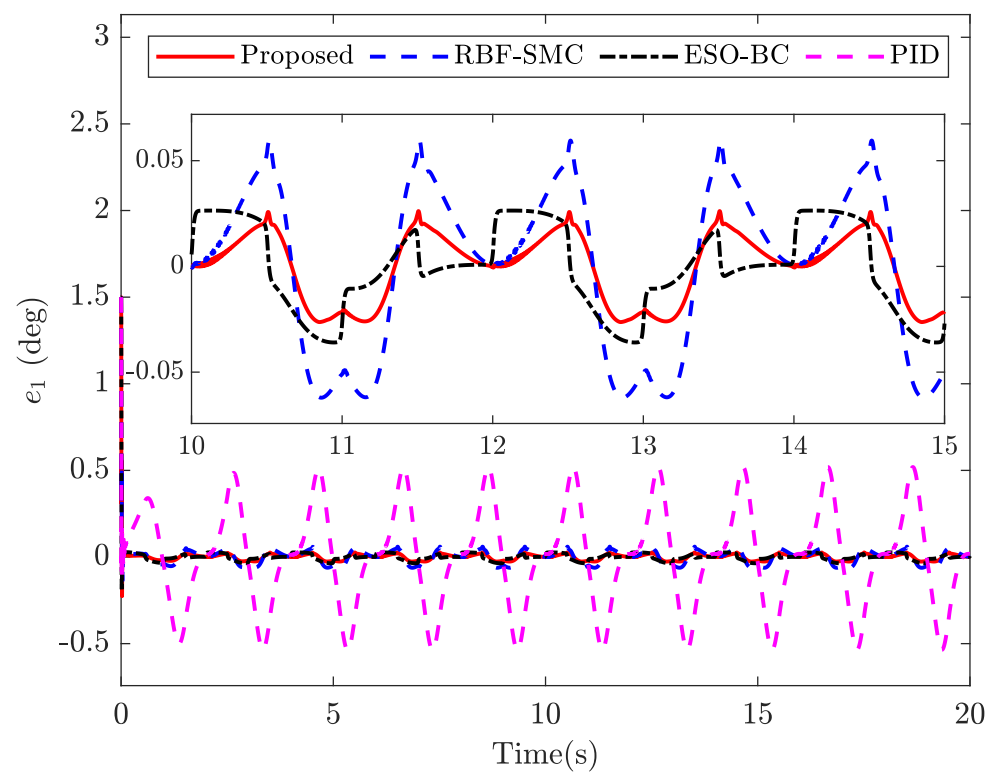

Figure 9. The tracking errors of the four controllers in fast-motion reference trajectory.

Table 4. Performance indexes in fast-motion reference trajectory of all considered controllers

\begin{tabular}{cccc}
\hline Controller & $\boldsymbol{M}_{\boldsymbol{e}}$ (Degree) & $\boldsymbol{\mu}_{\boldsymbol{e}}$ (Degree) & $\sigma_{\boldsymbol{e}}$ (Degree) \\
\hline Proposed Controller & 0.0263 & 0.0138 & 0.0086 \\
RBF-SMC Controller & 0.0621 & 0.0331 & 0.0203 \\
ESO-BC Controller & 0.0360 & 0.0151 & 0.0117 \\
PID Controller & 0.5364 & 0.2287 & 0.1843 \\
\hline
\end{tabular}

The simulation results of the two case studies demonstrate the superiorities and robustness against disturbances and uncertainties of the proposed control mechanism compared with others in achieving high-accuracy tracking performance for the EHSS without a chattering phenomenon. Although the use of the RBF NNs to cope with unstructured uncertainties is efficient, a number of parameters need to be carefully tuned to obtain better performance, which impedes its applications. Additionally, the suitability of the selection of radial basis function type when employing the RBF NNs to approximate unknown functions requires further careful investigation. Moreover, expert knowledge about the EHSS dynamics is also needed when designing the structure of NNs. Finally, the effectiveness of the adopted DOBs to compensate for the effects of disturbances on the controlled systems depends on the accuracy of the system state derivative calculation based on the Levant's differentiator, 
whose parameters are required to be meticulously selected to make the tradeoff between the computational precision and robustness against measurement noises.

\section{Conclusions}

In this paper, an adaptive robust control for the EHSS with full model uncertainties and disturbances based on RBF NNs and NN-based DOBs was proposed. For the first time, the combination of DOBs and RBF NNs was developed to effectively deal with both disturbances and completely unknown dynamics. In addition, the employment of the excessive switching gain of the controller was avoided; hence, the chattering issue was efficiently eliminated. The system stability was successfully proven by using the Lyapunov theory. The high-accuracy tracking performance achieved with several case studies demonstrated the effectiveness of the recommended control approach. In addition, the proposed method can be considered as a new framework dealing with control systems with limited knowledge about the system dynamics. Sophisticated control laws and experiments on a real test-bench will be considered in further studies.

Author Contributions: K.K.A. was the supervisor, providing funding and administrating the project, and he reviewed and edited the manuscript. H.V.D. checked the manuscript and discussed and supported the design of the proposed algorithm. M.H.N. performed the investigation, proposed the methodology, analysis, and validations by using the MATLAB/Simulink software for simulations, and wrote the original manuscript. All authors have read and agreed to the published version of the manuscript.

Funding: This research was supported by Basic Science Program through the National Research Foundation of Korea (NRF) funded by the Ministry of Science and ICT, South Korea (NRF 2020 R1A2B5B03001480) and this work was supported by "Regional Innovation Strategy (RIS)" through the National Research Foundation of Korea (NRF) funded by the Ministry of Education (MOE) (2021RIS-003).

Institutional Review Board Statement: Not applicable.

Informed Consent Statement: Not applicable.

Data Availability Statement: Not applicable.

Conflicts of Interest: The authors declare no conflict of interest.

\section{References}

1. Truong, H.V.A.; Trinh, H.A.; Ahn, K.K. Safety Operation of n-DOF Serial Hydraulic Manipulator in Constrained Motion with Consideration of Contact-Loss Fault. Appl. Sci. 2020, 10, 8107. [CrossRef]

2. Sun, C.; Fang, J.; Wei, J.; Hu, B. Nonlinear Motion Control of a Hydraulic Press Based on an Extended Disturbance Observer. IEEE Access 2018, 6, 18502-18510. [CrossRef]

3. Luo, C.; Yao, J.; Chen, F.; Li, L.; Xu, Q. Adaptive Repetitive Control of Hydraulic Load Simulator with RISE Feedback. IEEE Access 2017, 5, 23901-23911. [CrossRef]

4. Choi, K.; Oh, J.; Kim, H.-S.; Han, H.-W.; Park, J.-H.; Lee, G.-H.; Seo, J.; Park, Y.-J. Experimental Study on the Dynamic Characteristics of Hydro-Pneumatic Semi-Active Suspensions for Agricultural Tractor Cabins. Appl. Sci. 2020, 10, 8992. [CrossRef]

5. Yao, Z.; Yao, J.; Sun, W. Adaptive RISE Control of Hydraulic Systems with Multilayer Neural-Networks. IEEE Trans. Ind. Electron. 2019, 66, 8638-8647. [CrossRef]

6. Das, J.; Mishra, S.K.; Saha, R.; Mookherjee, S.; Sanyal, D. Nonlinear modeling and PID control through experimental characterization for an electrohydraulic actuation system: System characterization with validation. J. Braz. Soc. Mech. Sci. Eng. 2017, 39, 1177-1187. [CrossRef]

7. Mintsa, H.A.; Venugopal, R.; Kenne, J.; Belleau, C. Feedback Linearization-Based Position Control of an Electrohydraulic Servo System with Supply Pressure Uncertainty. IEEE Trans. Control Syst. Technol. 2012, 20, 1092-1099. [CrossRef]

8. Deng, W.; Yao, J.; Wang, Y.; Yang, X.; Chen, J. Output feedback backstepping control of hydraulic actuators with valve dynamics compensation. Mech. Syst. Signal Process. 2021, 158, 107769. [CrossRef]

9. Ahn, K.K.; Nam, D.N.C.; Jin, M. Adaptive Backstepping Control of an Electrohydraulic Actuator. IEEE/ASME Trans. Mechatron. 2014, 19, 987-995. [CrossRef]

10. Edwards, C.; Spurgeon, S. Sliding Mode Control: Theory and Applications; CRC Press: Boca Raton, FL, USA, 1998.

11. Khalil, H.K. Nonlinear Systems; Patience Hall: Hoboken, NJ, USA, 2002. (In English) 
12. Sun, G.; Wu, L.; Kuang, Z.; Ma, Z.; Liu, J. Practical tracking control of linear motor via fractional-order sliding mode. Automatica 2018, 94, 221-235. [CrossRef]

13. Ma, Y.; Li, D.; Li, Y.; Yang, L. A Novel Discrete Compound Integral Terminal Sliding Mode Control with Disturbance Compensation for PMSM Speed System. IEEE/ASME Trans. Mechatron. 2021, early access. [CrossRef]

14. Ma, Y.; Li, Y. Active Disturbance Compensation Based Robust Control for Speed Regulation System of Permanent Magnet Synchronous Motor. Appl. Sci. 2020, 10, 709.[CrossRef]

15. Utkin, V. Variable structure systems with sliding modes. IEEE Trans. Autom. Control 1977, 22, 212-222. [CrossRef]

16. Jelali, M.; Kroll, A. Hydraulic Servo-Systems: Modelling, Identification and Control; Springer Science \& Business Media: Berlin/Heidelberg, Germany, 2012.

17. Truong, L.V.; Huang, S.D.; Yen, V.T.; Cuong, P.V. Adaptive Trajectory Neural Network Tracking Control for Industrial Robot Manipulators with Deadzone Robust Compensator. Int. J. Control Autom. Syst. 2020, 18, 2423-2434. [CrossRef]

18. Zhang, Y.; Kim, D.; Zhao, Y.; Lee, J. PD Control of a Manipulator with Gravity and Inertia Compensation Using an RBF Neural Network. Int. J. Control Autom. Syst. 2020, 18, 3083-3092.[CrossRef]

19. Chen, Z.; Huang, F.; Sun, W.; Gu, J.; Yao, B. RBF-Neural-Network-Based Adaptive Robust Control for Nonlinear Bilateral Teleoperation Manipulators with Uncertainty and Time Delay. IEEE/ASME Trans. Mechatron. 2020, 25, 906-918. [CrossRef]

20. Li, T.; Duan, S.; Liu, J.; Wang, L.; Huang, T. A Spintronic Memristor-Based Neural Network with Radial Basis Function for Robotic Manipulator Control Implementation. IEEE Trans. Syst. Man Cybern. Syst. 2016, 46, 582-588. [CrossRef]

21. Yang, W.; Meng, F.; Meng, S.; Man, S.; Pang, A. Tracking Control of Magnetic Levitation System Using Model-Free RBF Neural Network Design. IEEE Access 2020, 8, 204563-204572. [CrossRef]

22. Li, X.; Fu, W.; Liu, L.; Wang, Y. Adaptive Dynamic Surface Control for Aircraft with Multiple Disturbances Based on Radial Basis Network. IEEE Access 2020, 8, 57709-57721.[CrossRef]

23. Liu, Y.J.; Zeng, Q.; Liu, L.; Tong, S. An Adaptive Neural Network Controller for Active Suspension Systems with Hydraulic Actuator. IEEE Trans. Syst. Man Cybern. Syst. 2020, 50, 5351-5360. [CrossRef]

24. Ruan, W.; Dong, Q.; Zhang, X.; Li, Z. Friction Compensation Control of Electromechanical Actuator Based on Neural Network Adaptive Sliding Mode. Sensors 2021, 21, 1508. [CrossRef] [PubMed]

25. Guo, Q.; Chen, Z. Neural adaptive control of single-rod electrohydraulic system with lumped uncertainty. Mech. Syst. Signal Process. 2021, 146, 106869. [CrossRef]

26. Fan, R.; Li, Y. An Adaptive Fuzzy Trajectory Tracking Control via Improved Cerebellar Model Articulation Controller for Electro-Hydraulic Shovel. IEEE/ASME Trans. Mechatron. 2021, 26, 2870-2880. [CrossRef]

27. Yao, J.; Deng, W. Active Disturbance Rejection Adaptive Control of Hydraulic Servo Systems. IEEE Trans. Ind. Electron. 2017, 64, 8023-8032. [CrossRef]

28. Wang, C.; Zhang, Z.; Wang, H.; Zhao, B.; Quan, L. Disturbance observer-based output feedback control of hydraulic servo system considering mismatched uncertainties and internal pressure dynamics stability. IET Control Theory Appl. 2020, 14, 1046-1056. [CrossRef]

29. Levant, A. Higher-order sliding modes, differentiation and output-feedback control. Int. J. Control 2003, 76, 924-941. [CrossRef]

30. Nguyen, M.H.; Dao, H.V.; Ahn, K.K. Active Disturbance Rejection Control for Position Tracking of Electro-Hydraulic Servo Systems under Modeling Uncertainty and External Load. Actuators 2021, 10, 20. [CrossRef]

31. Truong, H.V.; Tran, D.T.; To, X.D.; Ahn, K.K.; Jin, M. Adaptive Fuzzy Backstepping Sliding Mode Control for a 3-DOF Hydraulic Manipulator with Nonlinear Disturbance Observer for Large Payload Variation. Appl. Sci. 2019, 9, 3290. [CrossRef]

32. Na, J.; Li, Y.; Huang, Y.; Gao, G.; Chen, Q. Output Feedback Control of Uncertain Hydraulic Servo Systems. IEEE Trans. Ind. Electron. 2020, 67, 490-500. [CrossRef]

33. Wang, B.; Zhang, N.; Ji, H. Study on Precise Displacement Control of a Miniature Hydraulic System via RBF-DOB. IEEE Access 2018, 6, 69162-69171. [CrossRef]

34. Cheng, Y.; Xu, B.; Lian, Z.; Shi, Z.; Shi, P. Adaptive Learning Control of Switched Strict-Feedback Nonlinear Systems with Dead Zone Using NN and DOB. IEEE Trans. Neural Netw. Learn. Syst. 2021, early access. [CrossRef]

35. Levant, A. Robust exact differentiation via sliding mode technique. Automatica 1998, 34, 379-384. [CrossRef]

36. Merritt, H.E. Hydraulic Control Systems; John Wiley \& Sons: Hoboken, NJ, USA, 1967

37. Yao, J.; Jiao, Z.; Ma, D.; Yan, L. High-Accuracy Tracking Control of Hydraulic Rotary Actuators with Modeling Uncertainties. IEEE/ASME Trans. Mechatron. 2014, 19, 633-641. [CrossRef]

38. Yao, J.; Jiao, Z.; Ma, D. Extended-State-Observer-Based Output Feedback Nonlinear Robust Control of Hydraulic Systems with Backstepping. IEEE Trans. Ind. Electron. 2014, 61, 6285-6293. [CrossRef]

39. Park, J.; Sandberg, I.W. Approximation and Radial-Basis-Function Networks. Neural Comput. 1993, 5, 305-316. [CrossRef]

40. Orr, M.J. Introduction to Radial Basis Function Networks; Technical Report; Center for Cognitive Science, University of Edinburgh: Edinburgh, UK, 1996. 